\title{
BER Evaluation of A Low Complexity Transmit Diversity Scheme and Its Application to MIMO-BICM
}

\author{
Pei Xiao ${ }^{1}$, Boon Kien $\mathrm{Khoo}^{2}$, Stéphane Y. Le Goff ${ }^{3}$, William Scanlon ${ }^{4}$ \\ ${ }^{1}$ Centre for Communication Systems Research (CCSR) \\ University of Surrey, Guildford, UK. E-mail: p.xiao@surrey.ac.uk \\ ${ }^{2}$ Cambridge Broadband Networks, Cambridge Business Park \\ Cambridge, UK. E-mail: BKienKhoo@cbnl.com \\ ${ }^{3}$ School of Electrical, Electronic and Computer Engineering \\ Newcastle University, UK. E-mail: stephane.le-goff@ncl.ac.uk \\ ${ }^{4}$ Institute of Electronics, Communications and Information Technology (ECIT) \\ Queens University Belfast, Belfast, UK. E-mail: w.scanlon@qub.ac.uk
}

\begin{abstract}
In this paper, we first provide a theoretical validation for a low-complexity transmit diversity algorithm which employs only one RF chain and a low-complexity switch for transmission. Our theoretical analysis is compared to the simulation results and proved to be accurate. We then apply the transmit diversity scheme to multiple-input and multiple-output (MIMO) systems with bit-interleaved coded modulation (BICM).
\end{abstract}

\section{INTRODUCTION}

MIMO systems have been considered as one of the most promising technologies for high speed wireless communications, since the use of multiple antennas provides a useful tool to improve the performance of wireless communication links. MIMO can be categorized into i) spatial multiplexing (SM) MIMO based systems; ii) diversity based MIMO systems. SM systems transmit independent data streams from multiple transmit antennas to increase the data rate [1], [2]. On the other hand, the basic idea of diversity based MIMO is to receive (receive diversity) or transmit (transmit diversity) the same information-bearing signals redundantly through different channels so that the transmission reliability is improved. Diversity refers to the existence of two or more signal paths that fade independently. This happens when the radio channel consists of several paths that are sufficiently separated in space, time and frequency. It is unlikely that independent channels will fade together. Therefore, the reliability of transmitting the same signal via different paths will be consequently improved.

The advantage of using the transmit diversity is to obtain diversity gain without using multiple antennas at the mobile station, which is limited in size. Transmit diversity based on orthogonal space-time block codes (OSTBC) [3], [4] have received considerable interests. In general, OSTBC systems can achieve full diversity gain with simple linear processing at the receiver and without the knowledge of the channel information at the transmitter. However, when the number of transmit antennas is greater than two, an OSTBC system will suffer a rate loss. A viable solution is antenna selection at the transmitter [5]. However, its main drawback is the requirement of the feedback information from receiver to transmitter.

In this paper, we present a low-complexity transmit diversity scheme for MIMO systems employing two transmit antennas. This technique makes use of only one RF chain and a lowcomplexity switch to select transmit antenna sequentially. Since RF chains would consume most of the power in a portable device, this low-complexity diversity scheme also results in significant power saving, which is highly desirable in practical wireless systems. A mathematical model is then developed to assess the bit error rate (BER) performance of the proposed transmit diversity scheme, which is the main contribution of this paper. We then discuss the combination of space-time coding and bit-interleaved coded modulation (BICM) systems to achieve both diversity and coding gains. $\mathrm{BICM}$ is a bandwidth-efficient coding technique based on serial concatenation of binary error correction coding (ECC), bit-by-bit interleaving, and $M$-ary modulation [6]. It is capable of achieving excellent error performance when a capacityapproaching code is employed. For example, when a rectangular QAM modulation is coupled with a turbo code, BICM achieves a similar error performance to that obtained with more complicated turbo-coded modulation systems [7], [8]. In addition to turbo codes, classical convolutional codes are good alternative due to their low complexity compared to the capacity-approaching codes [6], [9]. The combination of BICM and MIMO (which is called MIMO-BICM) has attracted considerable attention recently due to its power and spectral efficiencies as well as robustness against fading. Furthermore, MIMO-BICM has been shown to outperform spacetime trellis coding in fast fading channels [10]. In this paper, we will demonstrate how the combination of the proposed transmit diversity scheme and MIMO-BICM improves power and spectral efficiencies in the investigated systems.

The notation used in this paper is summarized as follows: $(\cdot)^{\mathcal{H}}$ denotes matrix/vector conjugate transpose, $(\cdot)^{\mathcal{T}}$ denotes matrix/vector transpose, $(\cdot)^{*}$ denotes matrix/vector and complex scalar conjugate, and $\mathrm{E}[\cdot]$ denotes the expectation 

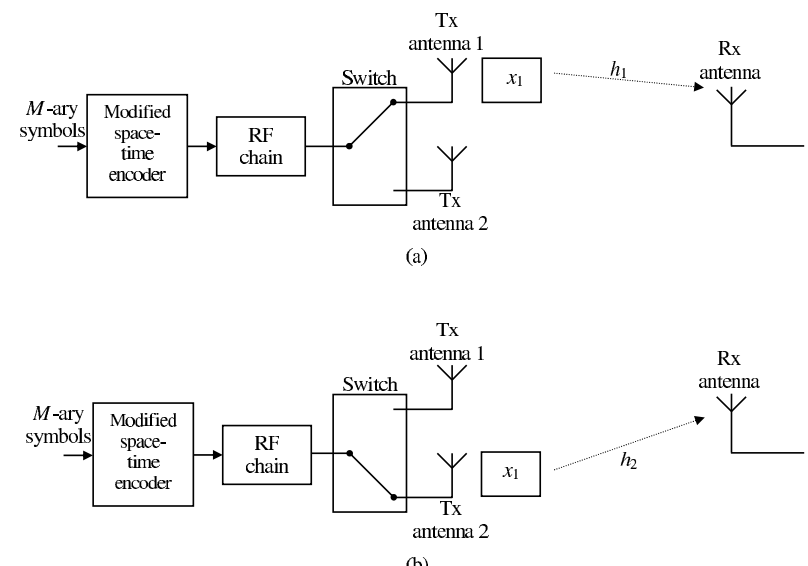

Fig. 1. Block diagram of the transmitter. (a) Proposed system at given time $t$; (b) Proposed system at given time $t+T$.

operation. Lower case bold letters, e.g., x, denote vectors; upper case bold letters, e.g., $\mathbf{X}$, denote matrices.

\section{Transmit Diversity Scheme And TheORETICAL ANALYSIS}

\section{A. Proposed Transmit Diversity Scheme}

In the proposed system shown in Fig. 1, two signals are transmitted sequentially from two transmit antennas using one $\mathrm{RF}$ chain and a low-complexity switch. At a given time $t$, antenna 1 is selected to transmit signal $x$. Then, at time $t+T$, antenna 2 is selected and the same signal $x$ is transmitted again. Since the proposed system uses only one RF chain and a low-complexity switch, a significant reduction in both complexity and size of the wireless devices can be achieved compared to the conventional space-time coded systems.

In the receiver, a buffer is employed in order to store the signal transmitted from antenna 1 before combining it with the signal transmitted from antenna 2 . The two received signals are given as follows: $y_{1}=h_{1} x+n_{1}, \quad y_{2}=h_{2} x+n_{2}$, where $y_{1}$ and $y_{2}$ are the received signals from antennas 1 and 2 , respectively. Once both signals from antenna 1 and antenna 2 are received, the maximal-ratio combining scheme is then applied to combine both received signals. Hence, the combined signal is given by

$$
z_{1}=\left(h_{1}^{*} y_{1}+h_{2}^{*} y_{2}\right) /\left(\left|h_{1}^{2}\right|+\left|h_{2}^{2}\right|\right)
$$

and finally sent to a maximum-likelihood detector. Note that the proposed system employs two transmit antennas and one receive antenna. It is, however, applicable to any configuration with two transmit antennas and an arbitrary number $n_{R}$ of receive antennas.

The decision statistic for the symbol $x$ in (1) can be simplified to

$$
z=x+w
$$

where $w \sim \mathcal{C N}\left(0, N_{w}\right), N_{w}=N_{0} / \mathcal{P}$, and $\mathcal{P}=\sum_{i=1}^{L}\left|h_{i}\right|^{2}$.

\section{B. Theoretical Analysis}

Next, we analyze the performance of the proposed scheme. For the 16-QAM modulated system, the bit error probability can be expressed as [11]

$$
P_{b}(\mathcal{P})=\frac{3}{4} Q\left(\sqrt{\frac{4 E_{b} \mathcal{P}}{5 N_{0}}}\right)+\frac{9}{8} Q^{2}\left(\sqrt{\frac{4 E_{b} \mathcal{P}}{5 N_{0}}}\right) .
$$

In order to obtain the average bit error probability for the proposed scheme, we need to average $P_{b}(\mathcal{P})$ over the distribution of the random variable $\mathcal{P}$, i.e.,

$$
\begin{aligned}
P_{b} & =\int_{0}^{\infty} P_{b}(\mathcal{P}) f(\mathcal{P}) d \mathcal{P}=\frac{3}{4} \underbrace{\int_{0}^{\infty} Q\left(\sqrt{\frac{4 \mathcal{P} E_{b}}{5 N_{0}}}\right) f(\mathcal{P}) d \mathcal{P}}_{\Psi} \\
& +\frac{9}{8} \underbrace{\int_{0}^{\infty} Q^{2}\left(\sqrt{\frac{4 \mathcal{P} E_{b}}{5 N_{0}}}\right) f(\mathcal{P}) d \mathcal{P}}_{\Omega},
\end{aligned}
$$

where $f(\mathcal{P})$ is the probability density function (PDF) of $\mathcal{P}$ and $\mathcal{P}=\sum_{i=1}^{L}\left|h_{i}\right|^{2}$. In our experiments, the amplitudes $\left|h_{i}\right|$ are characterized by a Rayleigh distribution. Therefore, the random variables $\left|h_{i}\right|^{2}$ follow a central Chi-square distribution with 2 degrees of freedom and characteristic function

$$
\psi_{\left|h_{i}\right|^{2}}(j v)=\left(1-j v \gamma_{i}\right)^{-1}
$$

where $\gamma_{i}=\mathrm{E}\left[\left|h_{i}\right|^{2}\right], \quad i=1, \ldots, L\left(L=2 n_{R}\right)$. In the case when the fading is assumed to be identically distributed with the same fading parameter, we have $\gamma_{i}=\gamma$ for all $i$. As a consequence of the statistical independence of $\left|h_{1}\right|^{2},\left|h_{2}\right|^{2}, \ldots,\left|h_{L}\right|^{2}$, the characteristic function of $\mathcal{P}$ can be expressed as

$$
\psi_{\mathcal{P}}(j v)=\prod_{i=1}^{L}(1-j v \gamma)^{-1}=(1-j v \gamma)^{-L} .
$$

Taking the inverse Fourier transform of (6), we obtain the PDF of $\mathcal{P}$ as [12]

$$
f(\mathcal{P})=\frac{1}{\gamma^{L}(L-1) !} \mathcal{P}^{L-1} \exp (-\mathcal{P} / \gamma), \quad \mathcal{P} \geq 0 .
$$

Substituting (7) into (4), we obtain the first integral as

$$
\Psi=\frac{1}{\gamma^{L}(L-1) !} \underbrace{\int_{0}^{\infty} Q\left(\sqrt{\frac{4 \mathcal{P} E_{b}}{5 N_{0}}}\right) \mathcal{P}^{L-1} \exp (-\mathcal{P} / \gamma) d \mathcal{P}}_{\Phi} .
$$

The integral in (8) can be solved as follows [13]:

$$
\Phi=\frac{1}{2} \gamma^{L}(L-1) !\left[1-\sum_{k=0}^{L-1} \nu\left(\frac{1-\nu^{2}}{4}\right)^{k}\left(\begin{array}{c}
2 k \\
k
\end{array}\right)\right],
$$

where

$$
\nu=\sqrt{\frac{2 E_{b} \gamma}{2 E_{b} \gamma+5 N_{0}}}
$$

Consequently,

$$
\Psi=\frac{1}{2}\left[1-\sum_{k=0}^{L-1} \nu\left(\frac{1-\nu^{2}}{4}\right)^{k}\left(\begin{array}{c}
2 k \\
k
\end{array}\right)\right],
$$


To solve the second integral in (4), we use the alternative representation of the Gaussian $Q$-function and its square [14]

$$
\begin{aligned}
Q(x) & =\frac{1}{\pi} \int_{0}^{\pi / 2} \exp \left(-\frac{x^{2}}{2 \sin ^{2} \theta}\right) d \theta \\
Q^{2}(x) & =\frac{1}{\pi} \int_{0}^{\pi / 4} \exp \left(-\frac{x^{2}}{2 \sin ^{2} \theta}\right) d \theta .
\end{aligned}
$$

With the above definition, $\Omega$ in (4) can be reformed as

$$
\begin{aligned}
\Omega & =\frac{1}{\pi} \int_{0}^{\pi / 4} \prod_{n=1}^{L} \exp \left(-\frac{g \gamma_{n}}{\sin ^{2} \theta}\right) d \theta \\
& =\frac{1}{\pi} \int_{0}^{\pi / 4} \prod_{n=1}^{L}\left[M_{\gamma_{n}}\left(-\frac{g}{\sin ^{2} \theta}\right)\right]^{L} d \theta,
\end{aligned}
$$

where $g=\frac{2 E_{b}}{5 N_{0}}$, and

$$
M_{\gamma_{n}}(s)=\int_{0}^{\infty} p\left(\gamma_{n}\right) \exp \left(s \gamma_{n}\right) d \gamma_{n}
$$

is the moment generating function (MGF) of the SNR per symbol $\gamma_{n}$ associated with the $n$-th channel. For Rayleigh fading channel, we have $M_{\gamma_{n}}(s)=\left(1-s \bar{\gamma}_{n}\right)^{-1}$ [14], where $\bar{\gamma}_{n}$ is the average SNR for the $n$-th channel. In the case when the fading is assumed to be identically distributed with the same fading parameter and the same average SNR $\gamma$ for all $L$ channels, we have $\bar{\gamma}_{n}=\gamma$ and $M_{\gamma_{n}}(s)=(1-s \gamma)^{-1}$. Therefore

$$
\begin{aligned}
\Omega & =\frac{1}{\pi} \int_{0}^{\pi / 4} \prod_{n=1}^{L}\left[M_{\gamma_{n}}\left(-\frac{g}{\sin ^{2} \theta}\right)\right]^{L} d \theta \\
& =\frac{1}{\pi} \int_{0}^{\pi / 4}\left(\frac{\sin ^{2} \theta}{\sin ^{2} \theta+g \gamma}\right)^{L} d \theta .
\end{aligned}
$$

A closed-form expression for the integral in (15) can be written according to [14] as

$$
\begin{aligned}
\Omega= & \frac{1}{\pi} \int_{0}^{\pi / 4}\left(\frac{\sin ^{2} \theta}{\sin ^{2} \theta+g \gamma}\right)^{L} d \theta \\
= & \frac{1}{4}-\frac{1}{\pi} \sqrt{\frac{g \gamma}{1+g \gamma}}\left\{\left(\frac{\pi}{2}-\tan ^{-1} \alpha\right) \sum_{k=0}^{L-1}\left(\begin{array}{c}
2 k \\
k
\end{array}\right) \frac{1}{[4(1+g \gamma)]^{k}}\right. \\
& \left.-\sin \left(\tan ^{-1} \alpha\right) \sum_{k=1}^{L-1} \sum_{i=1}^{k} \frac{T_{i k}}{(1+g \gamma)^{k}}\left[\cos \left(\tan ^{-1} \alpha\right)\right]^{2(k-i)+1}\right\},
\end{aligned}
$$

where $g \gamma=\frac{2 E_{b} \gamma}{5 N_{0}}, \alpha=\sqrt{g \gamma /(1+g \gamma)}$, and

$$
T_{i k}=\frac{\left(\begin{array}{c}
2 k \\
k
\end{array}\right)}{\left(\begin{array}{c}
2(k-i) \\
k-i
\end{array}\right) 4^{i}[2(k-i)+1]} .
$$

Combining (4), (11), and (16), yields the final expression for the average bit error probability of the proposed scheme as

$$
\begin{aligned}
P_{b} & =\frac{3}{8}\left[1-\sum_{k=0}^{L-1} \nu\left(\frac{1-\nu^{2}}{4}\right)^{k}\left(\begin{array}{c}
2 k \\
k
\end{array}\right)\right]+\frac{9}{32}-\frac{9}{8 \pi} \sqrt{\frac{g \gamma}{1+g \gamma}} . \\
& \left\{\left(\frac{\pi}{2}-\tan ^{-1} \alpha\right) \sum_{k=0}^{L-1}\left(\begin{array}{c}
2 k \\
k
\end{array}\right) \frac{1}{[4(1+g \gamma)]^{k}}\right. \\
& \left.-\sin \left(\tan ^{-1} \alpha\right) \sum_{k=1}^{L-1} \sum_{i=1}^{k} \frac{T_{i k}}{(1+g \gamma)^{k}}\left[\cos \left(\tan ^{-1} \alpha\right)\right]^{2(k-i)+1}\right\},
\end{aligned}
$$

where $\nu$ and $T_{i k}$ are given by (10) and (17), respectively.

\section{Performance Analysis in Presence of Spatial Correlations}

We have ignored the spatial correlations between multiple antennas in the above analyses. However, in real propagation environments, MIMO channels are correlated to a certain extend due to limited antenna element separation, geometry of array and surrounding objects in the near field of antenna elements, etc. [15]. Antenna correlation is defined as the envelope correlation coefficient between signals received at an antenna element, i.e.,

$$
\begin{aligned}
\rho & =\left|\frac{\mathrm{E}\left\{\left(h_{i}-E\left\{h_{i}\right\}\right)\left(h_{m}-E\left\{h_{m}\right\}\right)^{*}\right\}}{\sqrt{\mathrm{E}\left\{\left|h_{i}-E\left\{h_{i}\right\}\right|^{2}\right\} \mathrm{E}\left\{\left|h_{m}-E\left\{h_{m}\right\}\right|^{2}\right\}}}\right| \\
& =\left|\frac{\operatorname{cov}\left(h_{i}, h_{m}\right)}{\sqrt{\operatorname{var}\left(h_{i}\right) \operatorname{var}\left(h_{m}\right)}}\right|,
\end{aligned}
$$

Finding $f(\mathcal{P})$ (the PDF of $\mathcal{P}$ in Equation (4)) is tedious in the presence of antenna correlation. To work around this problem, we form a channel vector $\mathbf{h}=\left[\begin{array}{llll}h_{1} & h_{2} & \ldots & h_{L}\end{array}\right]^{T}$. Let the MIMO channel gains be represented by correlated complex Gaussian random variables. The joint PDF of $\mathbf{h}$ is determined by its mean vector $\mathbf{m}_{\mathbf{h}}$ and its covariance matrix $\mathbf{R}=\mathrm{E}\left\{\left[\mathbf{h}-\mathbf{m}_{\mathbf{h}}\right]\left[\mathbf{h}-\mathbf{m}_{\mathbf{h}}\right]^{\mathcal{H}}\right\}$, i.e.,

$p(\mathbf{h})=\frac{1}{(2 \pi)^{L}(\operatorname{det} \mathbf{R})^{1 / 2}} \exp \left[-\frac{1}{2}\left(\mathbf{h}-\mathbf{m}_{\mathbf{h}}\right)^{\mathcal{H}} \mathbf{R}^{-1}\left(\mathbf{h}-\mathbf{m}_{\mathbf{h}}\right)\right]$.

Note that the antenna correlation is taken into account in the covariance matrix $\mathbf{R}$. Since $\mathcal{P}=\mathbf{h}^{\mathcal{H}} \mathbf{h}$, Eq. (4) for the proposed scheme can be reformed as

$$
\begin{aligned}
P_{b} & =\int_{0}^{\infty} P_{b}(\mathcal{P}) f(\mathcal{P}) d \mathcal{P}=\frac{3}{4} \int_{\mathbf{h}} Q\left(\sqrt{\frac{4 \mathbf{h}^{\mathcal{H}} \mathbf{h} E_{b}}{5 N_{0}}}\right) f(\mathbf{h}) d \mathbf{h} \\
& +\frac{9}{8} \int_{\mathbf{h}} Q^{2}\left(\sqrt{\frac{4 \mathbf{h}^{\mathcal{H}} \mathbf{h} E_{b}}{5 N_{0}}}\right) f(\mathbf{h}) d \mathbf{h} .
\end{aligned}
$$

The conventional definition of the Q-function is given by $Q(x)=\int_{x}^{\infty} \frac{1}{\sqrt{2 \pi}} \exp \left(-\frac{y^{2}}{2}\right) d y$, which is substituted into (21) to derive the average bit error probability. However, the analysis is difficult to perform in this way since the argument $x$ appears in the lower limit of the integral. The problem can be tackled by using the alternative definite integral form for the Q-function shown in (12). To analyze the performance of the proposed scheme with antenna correlations, we also need to use the alternative definition of the squared Q-function expressed by (13). Substituting (12) and (13) into (21), the 
average bit error probability for the proposed scheme can be obtained according to [16] as

$$
\begin{aligned}
P_{b} & =\frac{3}{4 \pi} \int_{0}^{\frac{\pi}{2}}\left[\operatorname{det}\left(\frac{\boldsymbol{\Gamma}}{\sin ^{2} \theta}+\mathbf{I}\right)\right]^{-1} d \theta \\
& +\frac{9}{8 \pi} \int_{0}^{\frac{\pi}{4}}\left[\operatorname{det}\left(\frac{\boldsymbol{\Gamma}}{\sin ^{2} \theta}+\mathbf{I}\right)\right]^{-1} d \theta
\end{aligned}
$$

where $\boldsymbol{\Gamma}=\mathbf{R} / \beta^{2}$, and $\beta=\sqrt{5 N_{0} / 2 E_{b}}$.

Proposition: In the absence of antenna correlation, Eq. (22) reduces to Eq. (18).

Proof:

In this case, $\boldsymbol{\Gamma}=\mathbf{R} / \beta^{2}=\frac{2 E_{b} \gamma}{5 N_{0}} \mathbf{I}=c \mathbf{I}$, where $c=$ $2 E_{b} \gamma /\left(5 N_{0}\right)$. Let us denote

$$
P_{a}=\frac{1}{\pi} \int_{0}^{\frac{\pi}{2}}\left[\operatorname{det}\left(\frac{\Gamma}{\sin ^{2} \theta}+\mathbf{I}\right)\right]^{-1} d \theta,
$$

and use the notation $\psi(\theta)=\frac{c}{\sin ^{2} \theta}+1$, Eq. (23) can be written as

$$
\begin{aligned}
P_{a} & =\frac{1}{\pi} \int_{0}^{\frac{\pi}{2}}\left[\operatorname{det}\left(\frac{\mathbf{\Gamma}}{\sin ^{2} \theta}+\mathbf{I}\right)\right]^{-1} d \theta \\
& =\frac{1}{\pi} \int_{0}^{\frac{\pi}{2}}\left(\frac{c}{\sin ^{2} \theta}+1\right)^{-L} d \theta \\
& =\frac{1}{\pi} \int_{0}^{\frac{\pi}{2}} \psi(\theta)^{-L} d \theta=\frac{1}{\pi \Gamma(L)} \int_{0}^{\frac{\pi}{2}} \frac{\Gamma(L)}{\psi(\theta)^{L}} d \theta
\end{aligned}
$$

where the Gamma function is defined according to [17, p. 884] as

$$
\Gamma(L)=x^{L} \int_{0}^{\infty} e^{-x t} t^{L-1} d t, \quad x, L>0 .
$$

Substituting (25) into (24), we obtain

$$
\begin{aligned}
P_{a} & =\frac{1}{\pi \Gamma(L)} \int_{0}^{\frac{\pi}{2}} \frac{\psi(\theta)^{L}}{\psi(\theta)^{L}} \int_{0}^{\infty} e^{-\psi(\theta) t} t^{L-1} d t d \theta \\
& =\frac{1}{\Gamma(L)} \int_{0}^{\infty}\left(\frac{1}{\pi} \int_{0}^{\frac{\pi}{2}} e^{-c t / \sin ^{2} \theta} d \theta\right) e^{-t} t^{L-1} d t .
\end{aligned}
$$

Based on the alternative definition of the Q-function in (12), the inner integral in (26) can be expressed as

$$
\frac{1}{\pi} \int_{0}^{\frac{\pi}{2}} e^{-c t / \sin ^{2} \theta} d \theta=\frac{1}{\pi} \int_{0}^{\frac{\pi}{2}} e^{\frac{-(\sqrt{2 c t})^{2}}{2 \sin ^{2} \theta}} d \theta=Q(\sqrt{2 c t}) .
$$

Substituting (27) into (26) yields

$$
\begin{aligned}
P_{a} & =\frac{1}{\Gamma(L)} \int_{0}^{\infty} Q(\sqrt{2 c t}) e^{-t} t^{L-1} d t \\
& =\frac{1}{\Gamma(L) \gamma^{L}} \int_{0}^{\infty} Q\left(\sqrt{\frac{2 c \mathcal{P}}{\gamma}}\right) e^{-\mathcal{P} / \gamma} \mathcal{P}^{L-1} d \mathcal{P} \\
& =\frac{1}{\gamma^{L}(L-1) !} \underbrace{\int_{0}^{\infty} Q\left(\sqrt{\frac{2 c \mathcal{P}}{\gamma}}\right) \mathcal{P}^{L-1} \exp (-\mathcal{P} / \gamma) d \mathcal{P}}_{\Xi} .
\end{aligned}
$$

The second equality in (28) is derived by assigning $t=$ $\mathcal{P} / \gamma$. The third equality in (28) holds since $\Gamma(L)=(L-$
1)! [12] for positive integer $L$. The integral in (28) can be solved using the following equation [13]

$$
\begin{aligned}
& \int_{0}^{\infty} Q(\sqrt{2 a x}) x^{L-1} \exp (-x / \gamma) d x \\
& =\frac{1}{2} \gamma^{L}(L-1) !\left[1-\sum_{k=0}^{L-1} \mu\left(\frac{1-\mu^{2}}{4}\right)^{k}\left(\begin{array}{c}
2 k \\
k
\end{array}\right)\right],
\end{aligned}
$$

where $\mu=\sqrt{a \gamma /(2+a \gamma)}$. Assigning $a=2 c / \gamma$, we obtain the integral in (28) as

$$
\Xi=\frac{1}{2} \gamma^{L}(L-1) !\left[1-\sum_{k=0}^{L-1} \nu\left(\frac{1-\nu^{2}}{4}\right)^{k}\left(\begin{array}{c}
2 k \\
k
\end{array}\right)\right],
$$

where $\nu=\sqrt{\frac{a \gamma}{2+a \gamma}}=\sqrt{\frac{c}{1+c}}=\sqrt{\frac{2 E_{b} \gamma}{2 E_{b} \gamma+5 N_{0}}}$.

Based on the above analysis, it can be easily shown that

$$
\begin{aligned}
& \frac{1}{\pi} \int_{0}^{\frac{\pi}{2}}\left[\operatorname{det}\left(\frac{\boldsymbol{\Gamma}}{\sin ^{2} \theta}+\mathbf{I}\right)\right]^{-1} d \theta \\
& =\frac{1}{2}\left[1-\sum_{k=0}^{L-1} \nu\left(\frac{1-\nu^{2}}{4}\right)^{k}\left(\begin{array}{c}
2 k \\
k
\end{array}\right)\right],
\end{aligned}
$$

which is the value of $\Psi$ shown in (11). The parameter $\nu$ is defined in (10).

Now, let us analyze the second term in (22). In the absence of antenna correlation, $\boldsymbol{\Gamma}=\mathbf{R} / \beta^{2}=\frac{2 E_{b} \gamma}{5 N_{0}} \mathbf{I}=c \mathbf{I}$, where $c=2 E_{b} \gamma /\left(5 N_{0}\right)$. We have

$$
\begin{aligned}
& \frac{1}{\pi} \int_{0}^{\frac{\pi}{4}}\left[\operatorname{det}\left(\frac{\boldsymbol{\Gamma}}{\sin ^{2} \theta}+\mathbf{I}\right)\right]^{-1} d \theta \\
& =\frac{1}{\pi} \int_{0}^{\frac{\pi}{4}}\left[\operatorname{det}\left(\frac{2 E_{b} \gamma}{5 N_{0} \sin ^{2} \theta}+1\right) \mathbf{I}\right]^{-1} d \theta \\
& =\frac{1}{\pi} \int_{0}^{\frac{\pi}{4}}\left(\frac{2 E_{b} \gamma}{5 N_{0} \sin ^{2} \theta}+1\right)^{-L} d \theta \\
& =\frac{1}{\pi} \int_{0}^{\frac{\pi}{4}}\left(\frac{c}{\sin ^{2} \theta}+1\right)^{-L} d \theta=\frac{1}{\pi} \int_{0}^{\frac{\pi}{4}}\left(\frac{\sin ^{2} \theta}{\sin ^{2} \theta+c}\right)^{L} d \theta
\end{aligned}
$$

which is exactly the value of $\Omega$ shown in (15) (note that $c=$ $\left.g \gamma=2 E_{b} \gamma /\left(5 N_{0}\right)\right)$, leading to the same solution expressed by (16). Based on (30) and (31), we can conclude that (22) reduces to (18) in the absence of antenna correlation.

\section{Transmit Diversity Scheme for Bit-Interleaved Coded Mod- ulation Systems}

MIMO technique provides an effective means of achieving high data rate transmission without increasing the total tranmission power or bandwidth for wireless systems. On the other hand, BICM is a bandwidth and power efficient coded modulation scheme. The combination of MIMO and BICM systems has attracted considerable attentions as it provide both diversity and coding gains for wireless communication systems in multipath fading channels [18] - [20]. In this section, we investigate the association of the the proposed transmit diversity scheme with BICM systems.

In the transmitter, the information sequence $\left\{b_{n}\right\}$ is first of all encoded by a rate- $R_{c}$ convolutional encoder. Then, the resulting encoded sequence $\left\{u_{n}\right\}$ is interleaved by a random 
interleaver in order to generate an interleaved sequence $\left\{v_{n}\right\}$, which is further fed into a mapper with Gray labelling. The function of the mapper is to map groups of $\log _{2} M$ bits into one of the $M$ complex symbols. In this case, we consider the system with $n_{T}=2$. The modulated symbols $\left\{x_{n}\right\}$ are subsequently gathered to form successive groups of $n_{T}=2$ symbols.

At the first signaling interval, symbol $x_{1}$ is transmitted from the first antenna, while at the second signaling interval, symbol $x_{2}$ is transmitted from antenna 2. Note that, in the proposed system, the transmitted symbol $x_{1}$ is equal to the transmitted symbol $x_{2}$. Therefore, the overall spectral efficiency, $\eta_{P}$, of the proposed system is given by

$$
\eta_{P}=\frac{R_{c} \times \log _{2} M}{2} \quad \mathrm{bit} / \mathrm{s} / \mathrm{Hz} .
$$

In the receiver, the MRC scheme is applied to combine the received signals. The combined signals serve as inputs to a demapper for soft-decision demapping. The demapper produces soft estimates of the transmitted bits, which are subsequently deinterleaved and decoded.

\section{NumericAl AND ANALYTiCAl Results}

The investigated MIMO system has 2 transmit antennas and 1 or 2 receive antennas. The transmit power is set to $n_{T} \sigma_{s}^{2}=2$, i.e., unit average transmit power is used for each transmitted symbol. We first assume uncorrelated Rayleigh fading channel and the channel matrix is normalized such that the average channel gain for each transmitted symbol is equal to unity. In this case, the value of $\gamma$ in Eqs. (10) and (18) is $\gamma=1 / n_{R}$.

In Fig. 2, we compare the simulation results with the analytical results which are obtained by evaluating Eq. (18) for the proposed system. Simulation results are averaged over a minimum of 10,000 channel realizations and after at least 100 errors have occurred. Close agreement between the simulation results and analytical results for all the antenna setups have been observed from the figures. This confirms the accuracy of our theoretical analyses.

Fig. 3 shows the performance comparison in $2 \times 1$ and $2 \times 2$ MIMO systems with different levels of spatial correlations ( $\rho=0.0,0.4,0.8)$. The results are derived by numerically evaluating the single finite-range integral in (22). The figure clearly shows that antenna correlation has a detrimental effect for the system since the performance worsens as the correlation coefficient $\rho$ increases. A moderate level of correlation can be tolerated without significant performance loss if we compare the results corresponding to $\rho=0.0$ and those obtained for $\rho=0.4$. However, the system performance degrades drastically in presence of high antenna correlations, e.g., when $\rho=0.8$. It is desirable to reduce the correlations in the practical MIMO systems, for example, by increasing the antenna spacing at the transmitter and at the receiver.

Fig. 4 shows the performance of the proposed scheme in MIMO-BICM systems. The numbers of antennas are chosen to be $n_{T}=n_{R}=2$. We use two convolutional codes with rate $1 / 2$. The first code has a generator polynomial $(5,7)$ and constraint length of 3 , whereas the second code has a generator polynomial $(133,171)$ and constraint length of 7 . The employed modulation scheme is 16-QAM. Furthermore,

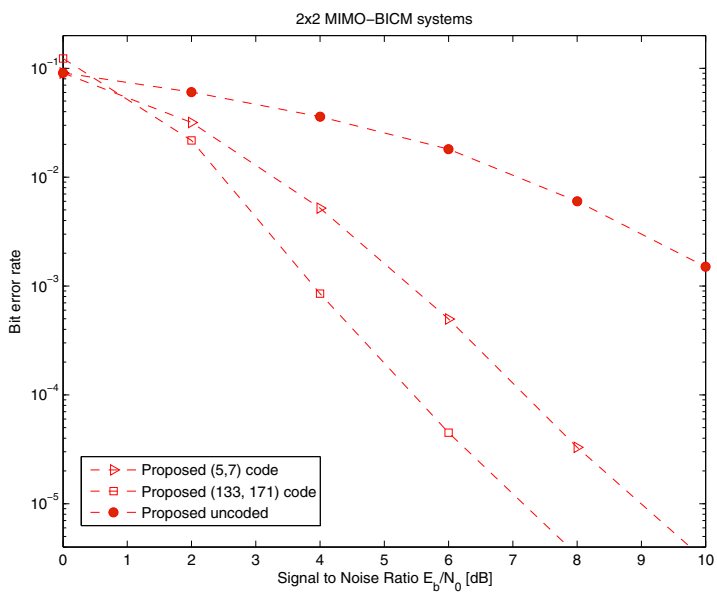

Fig. 4. Performance of the proposed MIMO-BICM system.

the size of interleavers are chosen to accommodate 1024 transmitted symbols, i.e. 4096 bits for the proposed scheme. The soft demapper is designed according to [7]. For comparison purposes, the performance of the uncoded systems is also provided in the figure, where one can see that channel coding significantly improves the system performance, even the system with a weak $(5,7)$ code performs much better than the uncoded system, the system performance improves as the channel code becomes stronger.

\section{CONCLUSIONS}

We provide a theoretical validation for a novel low complexity MIMO transmit diversity scheme. The theoretical analysis is verified by the simulation results and proved to be accurate. We then apply different diversity schemes to MIMO-BICM systems. Considering the fact that the proposed scheme has simple transmitter and receiver structure (i.e. less RF chains) and low space-time decoding complexity, it is therefore a viable solution for practical wireless communication systems. It also should be noted that the proposed scheme can be applied to wireless systems in order to obtain diversity gain, rather than capacity enhancement.

\section{REFERENCES}

[1] G. Foschini, "Layered space-time architecture for wireless communication in a fading environment when using multiple antennas". Bell Labs. Tech. J, vol. 1, no. 2, pp. 41-59, 1996.

[2] G. Foschini, G. Golden, R. Valenzuela, P. Wolniansky, "Simplified processing for high spectral efficiency wireless communication employing multi-element arrays". IEEE Journal on Selected Areas in Communications, vol. 17, no. 11, pp. 1841-1852, Nov. 1999.

[3] A. Alamouti, "A simple transmit diversity technqiue for wireless communications," IEEE J. Select. Areas in Commun., vol. 16, no. 8, pp. 1451-1458, Oct. 1998.

[4] V. Tarokh, H. Jafarkhani, and A. R. Calderbank, "Space-time block codes from orthogonal designs," IEEE Trans. Inform. Theory, vol. 45, no. 5, pp. 1456-1467, July 1999.

[5] S. Sanayei and A. Nosratinia, "Antenna selection in MIMO systems," IEEE Commun. Mag., vol. 42, no. 10, pp. 68-73, Oct. 2004.

[6] G. Caire, G. Taricco, E. Biglieri, "Bit-interleaved coded modulation". IEEE Trans. Inform. Theory, vo. 44, no. 3, pp. 927-946, May 1998.

[7] S. Y. Le Goff, A. Glavieux, and C. Berrou, "Turbo codes and high spectral efficiency modulation,” in Proc. IEEE ICC, pp. 645-649, May 1994. 


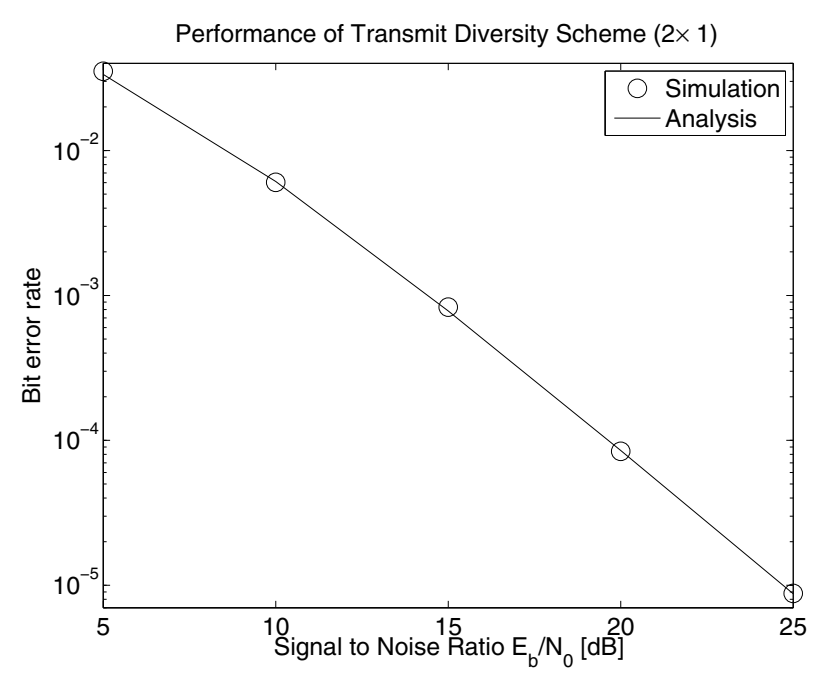

(a) $2 \times 1$ system.

Fig. 2. Analytical results vs simulation results.

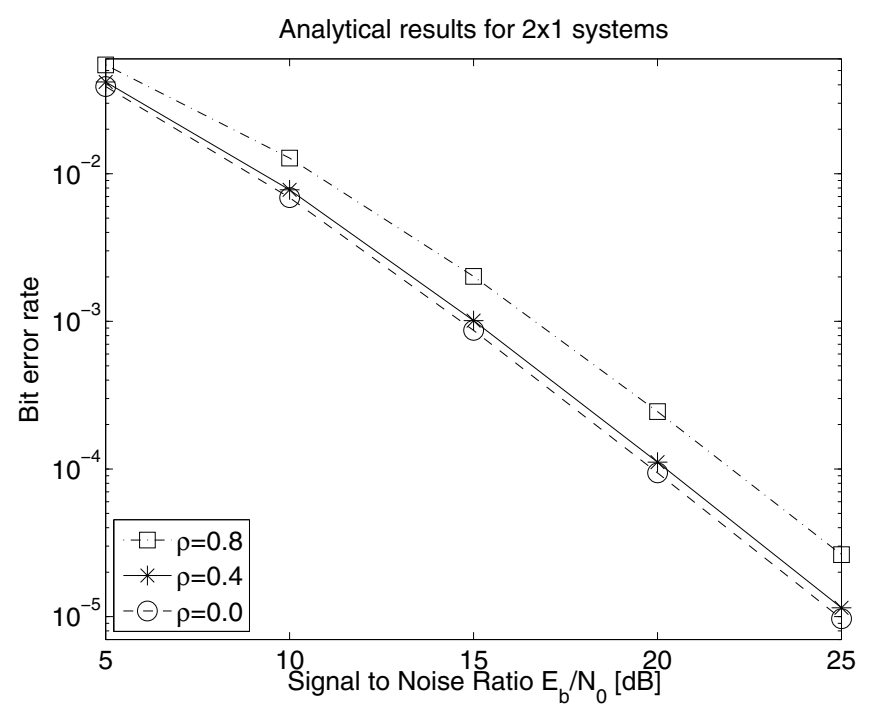

(a) $2 \times 1$ system.

Fig. 3. Performance analysis in presence of antenna correlations.

[8] A. Barbulescu, W. Farell, P. Gray, M. Rice, "Bandwidth-efficient turbo coding for high-speed mobile satellite communications". Proc. Int. Symp. Turbo Codes and Related Topics, pp. 119-126, Sept. 1997.

[9] S. Y. Le Goff, "Signal constellations for bit-interleaved coded modulation". IEEE Trans. Inform. Theory, vo. 49, no. 1, pp. 307-313, Jan. 2003.

[10] S. Muller-Weinfurtner, "Coding approaches for multiple antenna transmission in fast fading and OFDM". IEEE Trans. Sig. Proc., vol. 50, pp. 2442-2450, Oct. 2002.

[11] P. Xiao, R. Carrasco. I. Wassell, "Bit error rate evaluation of linear equalization in BFWA systems". International Journal of Communication Systems, vol. 20, no. 2, pp. 235-246, 2007.

[12] J. Proakis, Digital Communications. 3rd edition, McGraw-Hill, 1995.

[13] P. Xiao, L. Barbero, M. Sellathurai, and T. Ratnarajah, "On the uncoded BER performance bound of the IEEE 802.16d channel," IEEE Sig. Proc. Lett., vol. 15, pp. 561-564, 2008.

[14] M. Simon and M. Alouini, Digital Communication over Fading Channels - A Unified Approach to Performance Analysis. John Wiley \& Sons, 2000.

[15] M. K. Ozdemir and E. Arvas, "Dynamics of spatial correlation and implications on MIMO systems," IEEE Commun. Mag., vol. 42, no. 6, pp. 514-519, June 2004

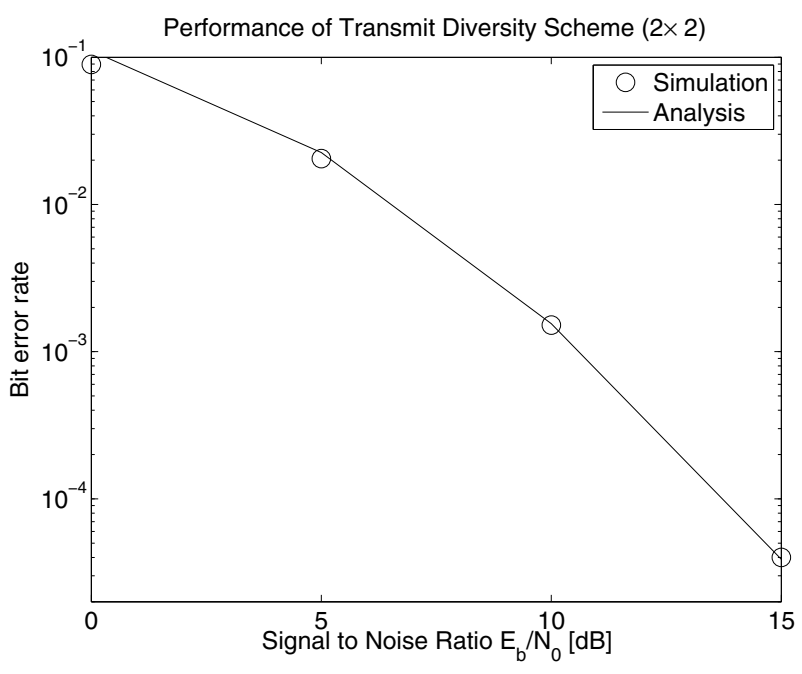

(b) $2 \times 2$ system.

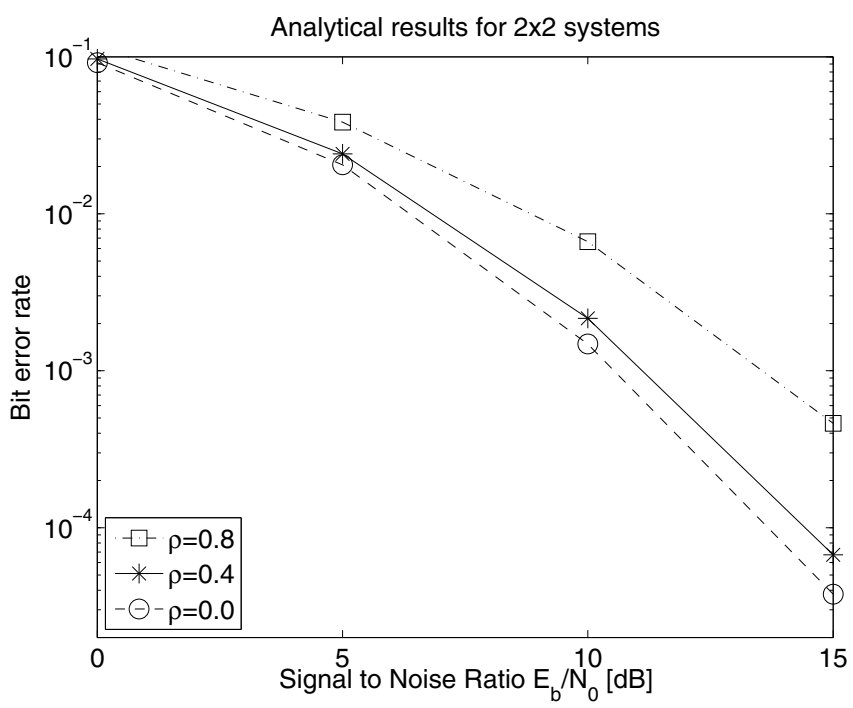

(b) $2 \times 2$ system.

[16] V. Veeravalli, "On performance analysis for signalling on correlated fading channels," IEEE Trans. Commun., vol. 49, no. 11, pp. 1879-1883, Nov. 2001.

[17] I. Gradshteyn and I. Ryzhik, Table of Integrals, Series, and Products. 6th edition, Academic Press, 2000.

[18] A. M. Tonello, "Space-time bit-interleaved coded modulation with an iterative decoding strategy," in Proc. VTC'00, Sept. 2000, vol. 1, pp. 473-478.

[19] Y. Huang and J. A. Ritcey, "Optimal constellation labeling for iteratively decoded bit-interleaved space time coded modulation," IEEE Trans. Inform. Theory, vol.51, pp. 1865-1871, May 2005.

[20] L. Zhao, J. Huber, and W. Gerstacker, "Design and analysis of bit interleaved coded space-time modulation," IEEE Trans. Commun., vol 56, pp. 904-914, June 2008. 